\title{
El sistema de relaciones territoriales entre las fortificaciones del Cilento interior. Una propuesta de estudio a través de SIG
}

The territorial relations system between the fortifications of the inner Cilento. A proposal for study through GIS

\author{
Maria Grazia Cianci $^{\text {a }}$, Sara Colaceci ${ }^{\text {b }}$, Francesca Paola Mondelli ${ }^{\text {c }}$ \\ ${ }^{a}$ Università degli Studi Roma Tre, Rome, Italy, mariagrazia.cianci@uniroma3.it \\ b Sapienza Università di Roma, Rome, Italy, sara.colaceci@uniroma1.it \\ ${ }^{\mathrm{c}}$ Università degli Studi Roma Tre, Rome, Italy, francescapaola.mondelli@uniroma3.it
}

\begin{abstract}
The landscape of the Cilento and Vallo di Diano National Park, south of Campania, is dotted with a wide variety of fortresses, castles, towers and all kinds of fortifications. The populations who settled there since the early Middle Ages have left traces of their presence through buildings that, from the initial purpose of independent defense of the individual settlement, have changed over time, especially in the Norman period, in a broader system and structured for the control of the territory. The diversity of the architecture that we still find today, determined by the different origins of the fortifications (sometimes Lombard, sometimes Norman, up to the coastal towers built in the Angevin-Aragonese period to defend against Saracen incursions), however, hides a complex system that connects these artifacts, creating a network that covers large portions of the territory. It is not easy today to recognize such relationships and rebuild this network that has in fact laid the foundations for the current layout of the Cilento landscape. The aim of the research is therefore to study, recognize and map this structure through the use of historical maps and direct relief. Starting from the portion of the territory related to the area of the Ancient Cilento, identified by the Monte della Stella Massif, we intend to start a mapping of the fortifications through GIS in order to provide the tools for the study of the geometries and territorial relations that were established between the different settlements, and how these were placed with respect to the surrounding territory. The use of the territorial information systems will also allow a systematic data collection that will open the way for a subsequent phase of survey and documentation of the artifacts scattered over the territory, through which it will be possible to create a typological abacus of the fortifications related to the different historical phases.
\end{abstract}

Keywords: Territorial system, fortifications, cultural landscape, GIS.

\section{Introducción. Marco del tema y del área de estudio}

El camino que conduce a la definición del valor del Parque Nacional del Cilento hoy en día, pasa por la comprensión de la estructura natural e histórica que a lo largo de los siglos ha determinado este territorio, y que aún hoy lo caracteriza. El ob- jetivo de nuestro estudio es investigar las fortificaciones del Cilento que comienzan a aparecer desde la época bizantina, y que cambian y se extienden simultáneamente a las luchas derivadas de las invasiones bárbaras, a escala territorial. 
Más que los objetos individuales, son las relaciones que se establecen entre ellos las que pretenden ser materia de nuestro interés, buscando en ellos los rasgos estructurales del paisaje histórico. Para ello, nos centraremos en el análisis de la zona del Monte Stella, ya que, según algunos estudios, el análisis de las relaciones visuales, históricas y culturales aún presentes en la actualidad, hacen de esta zona (Fig. 1) en sus límites más estrechos, el objeto de estudio más propio y característico de la conformación del antiguo Cilento. Nuestro interés, además, recae aquí por su importancia histórica ligada al sistema de fortificaciones, habiendo acogido la sede de la Baronía del Cilento, como veremos más adelante.

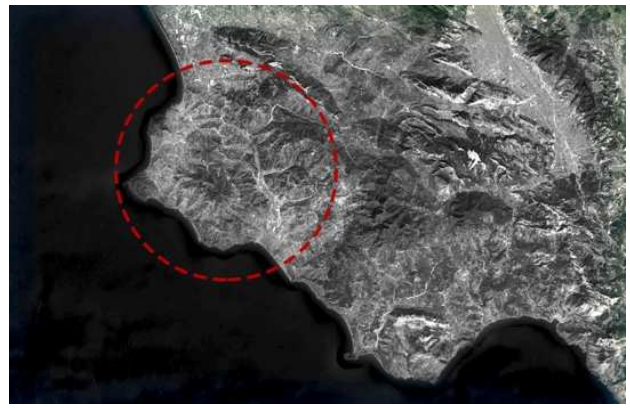

Fig. 1. Marco del area de studio.

\section{La estructura territorial. Relaciones entre el sistema de fortificaciones y la morfología del territorio}

Para analizar esta densa red de fortalezas, castillos y torres, es necesario ante todo tener en cuenta la conformación del territorio del Cilento, y más concretamente su estructura morfológica y geológica. Como se resume en el Plan de Paisaje, "la estructura del paisaje del Cilento está marcada por cursos fluviales muy diferentes (Alento, Calore, Lambro, Mingardo, Bussento) que entrecruzan numerosas cadenas montañosas y de colinas que son geomorfológicamente muy características. La franja costera está formada por la vista al mar de algunos de estos sistemas montañosos y las desembocaduras de los ríos, que sólo en el caso del Alento, abren grandes zonas de valle" (p. 35). Además, la doble naturaleza geológica del territorio ha generado estructuras morfológicas diferentes, a veces más suaves y accidentadas, a veces más duras y montañosas. Si la zona del Monte Stella y la llanura de Alento se caracteriza por el llamado Flysch del Cilento, es decir, una estratigrafía rocosa definida por una parte inferior de caliza, marga, arcilla y un conglomerado arenoso en la parte superior, los complejos montañosos interiores como Alburni, Cervati, Monte Bulgheria, etc., tienen un carácter calcáreo que da lugar a un paisaje modelado por las formas kársticas y por la aspereza de las vertientes.

Ya que las fortificaciones no tenían otra finalidad que la de implantar artificialmente una estructura defensiva cuyo papel ya estaba en parte cumplido por la dureza del territorio en el que las poblaciones buscaban refugio, remontando desde la costa hacia el interior, queda claro, por tanto, que el conocimiento de la morfología del territorio es fundamental para el análisis de las estructuras defensivas. No es casualidad, en efecto, si a lo largo de la historia, siguiendo las fases que dieron forma a la estructura del paisaje del Cilento, se hayan alternado periódicamente momentos en los que los asentamientos ocuparon la montaña y otros en los que se concentraron en la franja costera. En la Edad Media, las ciudades costeras, amenazadas por las incursiones bárbaras y sarracenas, fueron abandonadas en favor de centros más aislados y protegidos por la morfología más dura de las montañas, donde las comunidades monásticas comenzaron a establecerse y que desempeñarían un papel muy importante en la historia de Cilento a partir de este período.

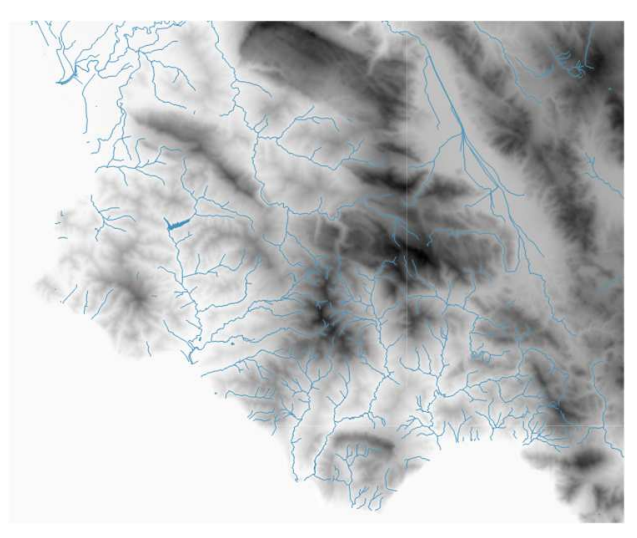

Fig. 2. Estudio de los sistemas topográficos e hidrográficos a través de software SIG. 
Esta será la planificación territorial que se mantendrá durante más tiempo, definiendo de hecho la conformación del paisaje del Cilento que ha llegado hasta nuestros días (Cianci, Mondelli, 2019). De hecho, incluso en épocas posteriores, cuando la necesidad será defender los frutos de la economía rural del bandolerismo generalizado, la conformación morfológica del territorio del Cilento, formado por montañas escarpadas, costas rocosas y llanuras pantanosas, constituirá una verdadera defensa natural que permitirá al Cilento mantenerse alejado de las inseguridades.

\section{Mapas aragoneses como punto de partida del estudio}

El interés por la morfología del territorio y el estudio de las características ambientales de la región del Cilento, también con vistas a un mejor control militar y administrativo, ya se expresaba en la antigüedad en algunos mapas aragoneses que describían el Principado de Citra. El Cilento, de hecho, durante la época del Reino de Sicilia, primero, y luego el Reino de Nápoles y las Dos Sicilias, formaba parte de la unidad administrativa del Principado Citra, correspondiendo aproximadamente a la zona que ahora representa la provincia de Salerno. Es gracias a las fuerzas económicas y a las técnicas avanzadas del reino, que se elaboraron mapas en el siglo XVIII que, teniendo en cuenta el margen de error de la época y las deformaciones que se pueden trazar, aplican un enfoque analítico muy riguroso, presentando de forma coreográfica el territorio. Esto significa que la descripción física del medio ambiente se asocia inmediatamente con detalles e informaciones de carácter antrópico, lo que pone de relieve el estrecho vínculo de interdependencia entre estos dos tipos de informaciones. En estos mapas, por tanto, abundan los topónimos que identifican a la vez elementos físicos como valles, montañas, sistemas hidrográficos, etc., e indicaciones sobre arquitecturas importantes, como santuarios, castillos, torres, ciudades amuralladas. Estos trabajos, encargados por el abad Ferdinando Galiani ${ }^{1}$, dan testimonio de una aproximación a la representación de la realidad que tiene como punto central la relación entre el entorno físico y los elementos antropogénicos, como signos de la actividad humana a lo largo de los siglos. Esto hace que los documentos no sean meras representaciones técnicas, sino la expresión de un pensamiento político que requiere el conocimiento del paisaje del Cilento, de las dinámicas que han guiado su desarrollo y de las redes de asentamientos que a lo largo del tiempo lo han estructurado, con el fin de asegurar su correcta administración. Precisamente por esta razón, el estudio de los mapas aragoneses ha sido de gran ayuda para nuestro trabajo. Las ciudades fortificadas, como por ejemplo Agropoli, están representadas aquí, diseñadas a vista de pájaro con sus murallas o castillo; las ruinas, los restos y las antigüedades están marcados; por último, se reporta el sistema de torres costeras que caracteriza la costa del Cilento. En cuanto a los centros fortificados, hay que decir que son los más numerosos. Hay que tener en cuenta, de hecho, que los mapas muestran la red de fortificaciones presentes en la época angevina (situación que se mantuvo casi inalterada incluso en la época aragonesa), y por lo tanto ya cargada de las estratificaciones temporales de las intervenciones defensivas más antiguas: desde los longobardos a los normandos, pasando por suevos y, sobre todo, por los angevines.
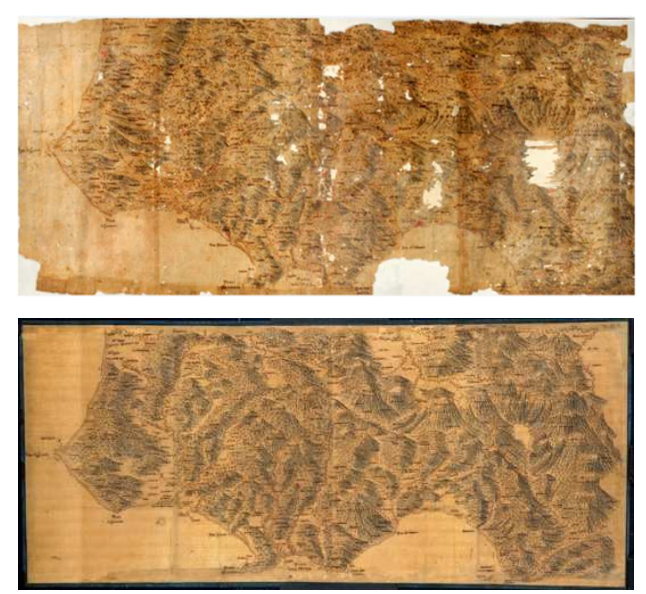

Fig. 3. El Principado Citra en los mapas aragoneses (siglo XVIII). Arriba: Archivio di Stato di Napoli. Abajo: Bibliothèque Nationale de France.

En particular, las zonas que, en los mapas, parecen mostrar una mayor importancia, son las relativas a los centros fortificados de Agropoli, Castellabate y Velia (Castello della Bruca). También 
se indica La Rocca, es decir, la actual Rocca Cilento, ciudad fortificada de especial interés e importancia para los fines de nuestro estudio, ya que es aquí donde, en el siglo XI, con el establecimiento del Actus Cilenti, el Cilento asume su propia connotación territorial y administrativa, o sea, la Baronia del Cilento. Si esto, en un primer momento, reconoce su sede en el Castellum Cilenti, una fortificación situada en la cima del Monte della Stella y de la que hoy quedan pocos vestigios, entonces se colocará en Rocca la sede de la Baronía, que bajo el poder de los señores feudales Sanseverino adquirirá cada vez más importancia.

\section{El uso de SIG para el reconocimiento de las relaciones visuales entre centros fortificados}

Partiendo de las reflexiones surgidas del estudio del enfoque analítico y técnico utilizado en los mapas aragoneses, al pasar a la realización de nuestro estudio mediante el uso de software SIG, intentamos primero crear un mapa que combinara las características del entorno físico con algunas de las principales huellas históricas antrópicas, con el fin de leer de forma completa, aunque esquemática, los rasgos distintivos del paisaje histórico de Cilento. Creímos conveniente componer el mapa distribuyendo los factores antrópicos en diferentes niveles, considerados esenciales para la lectura del territorio:

- Red histórica de carreteras

- Red de carreteras de origen romano

- Centuriazioni romanas

- Centros históricos

- Yacimientos Arqueológicos

Estos niveles, superpuestos a la orografía del suelo obtenida a través del archivo DEM, a los principales vectores que conforman el sistema hidrográfico y a los mapas históricos georeferenciados a través del software, han constituido la primera base sobre la que ir a trabajar, más tarde, para el reconocimiento y el mapeo de la red de fortificaciones diseminadas por el territorio.

Muy importante en este panorama general es el reconocimiento de los asentamientos, primero griegos y luego romanos, de Pestum, al norte, y de Velia, al sur. Su ubicación, situada en ambos casos en correspondencia de los valles fluviales (Sele y Solofrone al norte, Alento al sur), se configuran como puertas territoriales del Antiguo Cilento, delimitando de alguna manera la zona del Monte Stella a través de las rutas históricas que sirvieron de enlace entre las dos ciudades.

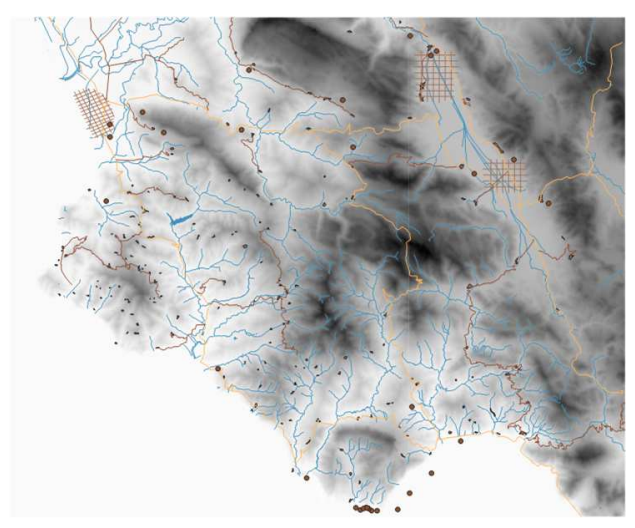

Fig. 4. Construcción del mapa base sobre la que se ha trabajado para la individuación de la red de fortificaciones.

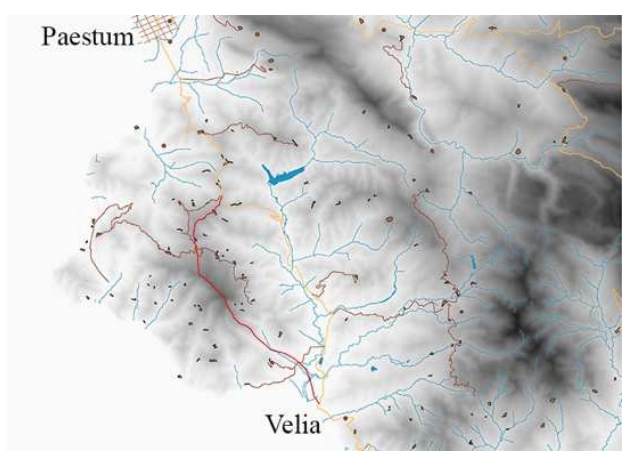

Fig. 5. Hipotesis reconstructiva de la ruta de cresta, en rojo, que pasa por el Monte della Stella, colegando las ciudades griegas-romanas de Paestum y Velia.

Sin embargo, más allá de las dos rutas principales que consisten en la ruta costera, al oeste, y la ruta del fondo del valle, al este, se cree que una conexión de gran importancia estaba constituida por la antigua ruta de la cresta principal que cruzaba el Monte Stella, pasando por la cima del Castellum Cilentí y por el centro fortificado de Rocca. Esta ruta, identificada por nosotros y trazada en el mapa SIG, es fundamental en cuanto a las relaciones visuales que se establecieron entre los centros 
de administración del poder de la Baronía del Cilento, y el territorio circundante. La reconstrucción de esta ruta, hoy desaparecida, se basó en el estudio de la orografía del Monte Stella y en la forma de los centros históricos que surgen a lo largo de la línea de la cresta, que aún hoy dejan huellas perfectamente legibles del antiguo camino. Desde un punto de vista visual y estratégico, esta ruta constituye la columna vertebral que estructura todo el paisaje. El centro fortificado de Rocca, situado a lo largo de esta línea de cresta principal, no sólo es el punto desde el que se puede controlar el territorio, sino que se convierte en una figura fuerte dentro de la escena, en relación de la cima del Monte Stella, y visible desde perspectivas lejanas, representando físicamente el poder. A través de esta ruta de cresta es posible, por tanto, trazar la relación entre los dos polos de Rocca Cilento y Castellum Cilenti, pero no sólo. Como muestran también los estudios de Pasquale del Duca, "Rocca y la cima del Monte della Stella están en relación visual con una zona que va mucho más allá de los límites del antiguo Cilento, hasta la llanura del Sele y el Monte Soprano al norte, y el Monte Gelbison al este, por lo que podemos creer que forman parte de un sistema de relaciones visuales mucho más amplio que el que existe dentro de este territorio". Cruzando los límites del Antiguo Cilento, por lo tanto, hemos tratado de identificar, a través de inspecciones, reconocimientos directos y análisis a través de herramientas satelitales, las relaciones visuales más amplias que se establecen entre el Castillo de Rocca Cilento y las otras fortificaciones importantes que se encuentran en el territorio. Se puede deducir una interesante línea visual, que alinea la sede de la Baronia del Cilento con la Rocca di Agropoli, al noroeste, y por lo tanto hacia el mar, mientras en la dirección opuesta, hacia el sureste, se puede ver a lo lejos la fortaleza de Novi Velia. Este último en particular es un vínculo importante, ya que Novi fue a su vez el hogar de una Baronía, la secular de Nobe, el centro de otra red de fortificaciones que se extendió aún más al sur, incluyendo Magliano Vetere, Gioi Cilento y $\mathrm{Cu}$ ccaro Vetere.

Según Ebner, en efecto, tras la caída del principado lombardo de Salerno en 1077, el territorio del Cilento se dividió en tres baronías importantes: la de la Abadía de Cava, la de los Sanseverino (44 casali), que tienen su sede en la localidad de

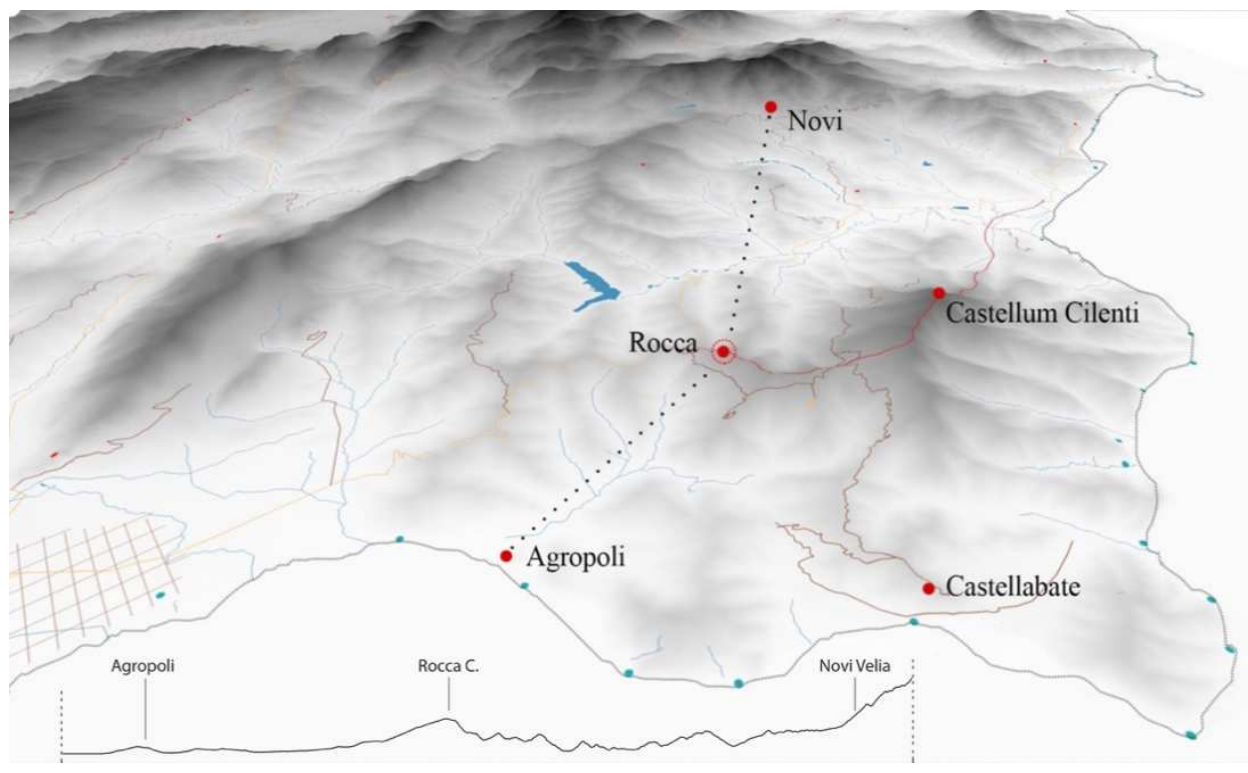

Fig. 6. Vista 3D y perfil territorial hechos a través de software SIG, el la que se marca la relación visual entre los centros fortificados de Rocca, Novi Velia y Agropoli. Se trazan también las fortezas cercanas de Castellabate y la de Castellum Cilenti, colocada sobre la misma línea de cresta de Rocca Se pueden ver, en azul, las torres costeras. 
Rocca Cilento, y la de Magnia di Novi, que incluye las tierras de Novi (13 casali), de Cuccaro (9 casali), de Gioi (10 casali), de Magliano (4 casali), e incluso de la ciudad de Monteforte, al norte. Por lo tanto, es interesante observar cómo, sobre la base de un sistema de relaciones principales constituido por las alineaciones de los lugares de poder, se injertaron más redes de control territorial en cada uno de los feudos, que, sin embargo, no configuraron estructuras cerradas, sino que fueron colocadas dentro de una macro-red que puede ser trazada en la estructura morfológica del territorio.

\section{Mapeo en SIG de la red de fortificaciones}

Para descubrir y rastrear a mano esta red de fortificaciones en el paisaje del Cilento, ha sido fundamental, en primer lugar, dedicarse a la identificación y clasificación de las estructuras arquitectónicas todavía presentes en el territorio, empezando por una recogida de datos que permitiera dar indicaciones al menos sobre la base de seis parámetros:

- Nombre propio de la fortaleza
- Tipo de fortificación

- Municipio en el que se ubica

- Estado de conservación

- Período de construcción

- División administrativa de referencia en el periodo histórico analizado

Siempre trabajando con software SIG, hemos creado un archivo shapefile que contiene toda la información relacionada con las fortificaciones, para luego trabajar en la construcción de una tabla de atributos a través de la cual sistematizar los datos. Esto nos permitió, más tarde, visualizar los puntos que dibujamos en el mapa de forma clasificada e informada, de acuerdo con los parámetros que atribuimos. La construcción de este archivo ha sido sin duda la parte más exigente del trabajo, hasta el día de hoy, ya que la investigación se encuentra todavía en una fase cognitiva de recogida de datos y catalogación, aunque hace referencia continua a una visión de conjunto y guarda las relaciones que surgen naturalmente ya durante esta primera fase.

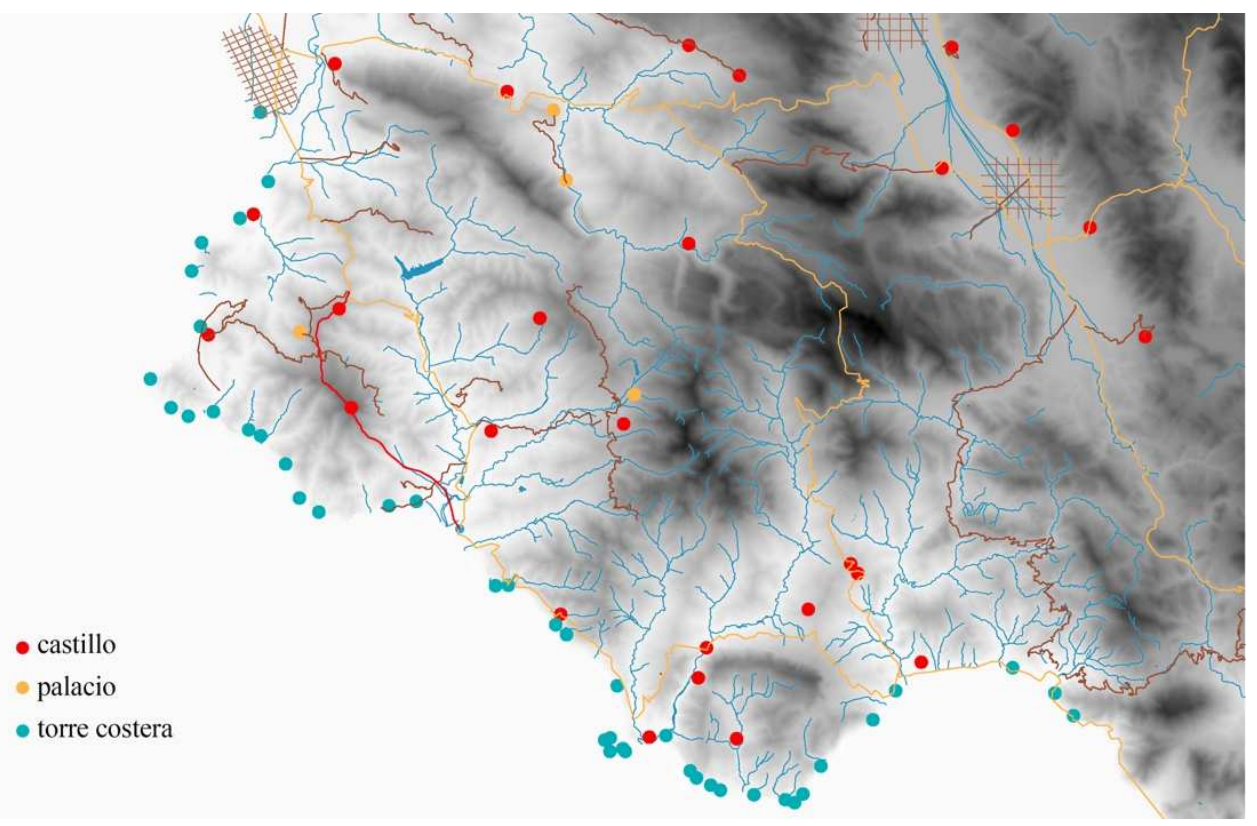

Fig. 7. Mapa de las fortificaciones distribuidas sobre todo el territorio del Cilento. Los diferentes colores evidencian la clasificación tipológica de las fortalezas: castillos, torres costeras, palacios fortificados. 
La recopilación y clasificación de los datos se ha realizado mediante la comparación de diferentes fuentes. Desde el estudio de los mapas históricos, que ya hemos mencionado, hasta los estudios previos de otros estudiosos, en particular los de Antonio Capano, que a su vez se refiere a los estudios fundamentales de Pietro Ebner y Fernando La Greca. Estos datos han sido luego cruzados por nosotros con los reportados por el Plan de Paque Nacional de Cilento y Vallo di Diano, disponibles en Open Data en la página oficial del Parque, y también, como ya se ha mencionado, con un conocimiento directo del territorio. El resultado es un esquema claro y sistemático, que por el momento contempla la clasificación de las fortalezas en tres niveles: castillos, palacios fortificados (sobre todo si las fuentes muestran que se levantan sobre los restos de castillos anteriores) y torres costeras.

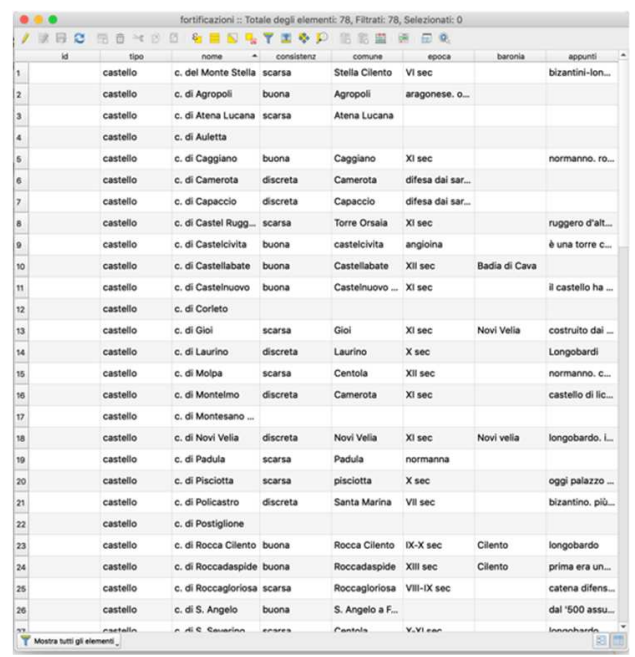

Fig. 8. El archivo SIG ha sido realizado clasificando las fortificaciones según diferentes parámetros.

La cuestión de las torres costeras sería, en realidad, un tema por derecho propio, que esta contribución no pretende tratar en profundidad. Sin embargo, parece necesario mencionarlo en aras de la exhaustividad, ya que la falta de estos elementos, dentro del mapa, proporcionaría una visión distorsionada e incompleta del contexto del Cilento, inaceptable si nuestra intención es estudiar las fortificaciones a escala territorial. Nuestro mapa muestra 42 torres costeras: desde la Torre Pestum, al norte, hasta la Torre de la Medianoche (Sapri), al sur, que hemos identificado como los límites de nuestra área de estudio. Este tipo de torres de observación en la costa del Cilento comenzaron a construirse ya en el siglo $\mathrm{X}$, y luego se multiplicaron en los períodos normando y suevo, con torres generalmente altas y cilíndricas. Sin embargo, fue en la época angevina cuando estas torres se convirtieron en un verdadero sistema compacto, distribuido uniformemente a lo largo de la costa. Esta es la estructura que encontramos descrita también en los mapas aragoneses que utilizamos para nuestro estudio.

\section{Conclusiones. Indicaciones sobre desarro- llos futuros}

El sistema de fortificaciones del interior del Cilento, investigado a escala territorial y teniendo en cuenta el aspecto paisajístico, es un tema de investigación amplio y complejo para el que es necesario recurrir a los valiosos estudios realizados en el pasado por historiadores, geógrafos y arqueólogos. Como arquitectos, nos acercamos al tema proponiendo una síntesis sistemática, con el objetivo de sistematizar el gran patrimonio que nos ha llegado, para trazar la estructura histórica que une los centros que aún hoy conforman el paisaje del interior del Cilento. El estudio de estas relaciones ofrece la oportunidad de investigar y sacar a la luz las geometrías invisibles que dibujan el paisaje histórico, las geografías inmateriales que han generado los asentamientos, ofreciendo la oportunidad de potenciar, a nivel programático, aquellas que son las relaciones visuales y panorámicas que vinculan el entorno natural del Parque con su arquitectura histórica. La investigación, como ya se ha mencionado, está llevando a cabo su primera fase de recopilación y catalogación de datos. Hasta ahora, el uso de software SIG ha sido útil para la creación de archivos informados en los que recoger los datos y las fuentes utilizadas; en la siguiente fase, se pretende utilizar SIG 3D para realizar análisis de puntos de vista basados en la morfología del terreno obtenida a través de los Modelos Digitales de Terrenos (MDT), con los que se determinará la visibilidad mutua entre la red de fortalezas que se encuentran en el territorio. Además, se pretende comprobar los resultados obtenidos a través de un 
análisis visibilidad: line of sight y viewshed. Un estudio tridimensional orientado al conocimiento y mejora del paisaje fortificado del antiguo Cilento.

\section{Notas}

${ }^{1}$ Se hicieron entre 1767 y 1769 . El primero, se conserva en la Colección de Plantas y Dibujos de los Archivos Estatales de Nápoles. El segundo, realizado por Giovanni Pontano, se conserva en la Biblioteca Nacional de París, y fue descrito y analizado por Fernando La Greca y Vladimiro Valerio en el libro Paisaje antiguo y medieval en los mapas aragoneses de Giovanni Pontano. Las tierras del Principado de Citra (2008).

\section{Bibliography}

Acocella, N. (1961). "Il Cilento dai Longobardi ai Normanni. Struttura amministrativa e Agricola", Rassegna Storica Salernitana, XXII, Napoli.

Almagià R. (1972). "Studi storici della cartografia napoletana" in Mazzetti, E. ed., Cartografia generale del mezzogiorno e della Sicilia, Edizioni Scientifiche Italiane, Napoli.

Anzani, G.; Mazzoleni, D. (1993). Il Cilento Antico. I luoghi e l'immaginario, Electa, Napoli.

Capano, A. (2014). "Castelli, palazzi baronali e borghi fortificati nel Cilento. Lineamenti storici”, Salternum, 32-33.

Cianci, M.G.; Mondelli, F.P. (2019). "Landscape development in Cilento National Park: the small towns of Monte Stella area between rural economy and tourism", in Atti congresso STC, Salerno.

Ebner, P. (1973). Storia di un feudo del Mezzogiorno. La baronia di Novi, Edizioni di Storia e Letteratura, Roma.

Greca, F. La; Valerio, V. (2008). Paesaggio antico e medievale nelle mappe aragonesi di Giovanni Pontano. Le terre del Principato Citra, Ogliastro Cilento.

Landi, F. (2014). "Paesaggi virtuali del passato. Cartografia storica, GIS e virtual landscaping: il caso dell'isola Palmaria", in Gallia, A. ed., Studi storico-cartografici. Dalla mappa al GIS, Genova.

Longley, P.A.; Goodchild, M.F.; Maguire, D.J.; Rhind, D.W. (2005). Geographic Information Systems and Science, John Wiley and Sons, Chichester.

PNCVD. (2010). Piano del Paesaggio, p. 35.

Santoro, L. (1992). "Il Sistema difensivo territoriale a seguito dell'unificazione normanna", in Notarangelo, A. ed., Torri e Castelli del mezzogiorno, I.Pi.Ge.T. - C.N.R., Giannini \& figli Ed., Napoli.

Visentin, B. (2016). "La rappresentazione dello spazio nel mezzogiorno aragonese: il Cilento antico", in Vitolo, G. ed., La rappresentazione dello spazio nel mezzogiorno aragonese: Le carte del principato Citra, Laveglia e Carlone s.a.s., Battipaglia.

Vitolo, G. (2013). "Governo del territorio e rappresentazione dello spazio nel Mezzogiorno aragonese", in Barone, G.; Esposito, A.; Frova, C., eds., Ricerca come incontro. Archeologi, paleografi e storici per Paolo Delogu, Roma.

Volpe, G. (1888). Notizie storiche delle antiche città e dei principali luoghi del Cilento, Ripostes, Salerno.

Vassalluzzo, M. (1969). Castelli, torri e borghi della costa cilentana, Linotypografia, Salerno. 